\title{
The Influence of Perceived School Importance on Adolescent Smoking Intensity ${ }^{*}$
}

\author{
Jill A. Nolan \\ Concord University, \\ Athens, WV USA
}

\author{
Traci Jarrett \\ West Virginia \\ University, \\ Morgantown, WV \\ USA
}

\author{
Laura Kurth \\ National Institute of \\ Occupational Safety \\ and Health (NIOSH), \\ Morgantown, WV \\ USA
}

\author{
Kim Horn \\ George Washington \\ University, \\ Washington DC, \\ USA
}

\author{
Geri A. Dino \\ West Virginia \\ University, \\ Morgantown, USA
}

\begin{abstract}
Smoking is currently one of the most detrimental causes of preventable chronic disease. Lower levels of education attainment are linked with poor health outcomes in adulthood. Further, adults who smoke generally have lower levels of education. Smoking in adolescence is linked with poor educational outcomes such as dropout and academic performance. Little is known about the influence of perceived academic importance on adolescent smoking intensity. Utilizing secondary data collected through the N-O-T (Not-On-Tobacco) intervention, this study explored the influence of perceived school importance, nicotine dependence, gender, race, and grade on smoking intensity in a sample $(N=197)$ of adolescent smokers seeking cessation. Results showed that perceived school importance $t(191)=-2.96, p<0.003$, nicotine dependence $t(191)=9.84, p<0.000$, grade $t(191)=3.54, p<0.00$, and gender $t(191)=2.52, p<0.02$ were all significant predictors of smoking intensity. Due to the established relationship between smoking and poor educational performance and the relationship between both smoking and education and poor health outcomes, these findings identify a need to emphasize the importance of educational attainment in intervention efforts.
\end{abstract}

Keywords: smoking cessation, adolescent risk behavior, education

Smoking continues to be one of the most detrimental yet preventable causes of chronic disease despite ongoing tobacco prevention and cessation efforts in the United States (Ezzati \& Lopez, 2003; Warren et al., 2006). Although adolescent tobacco use shows a modest decrease, 2009 national data indicate that $46 \%$ of 9 th

\footnotetext{
* Acknowledgements: The authors would like to acknowledge the Translational Tobacco Reduction Research Program (T2R2) and the West Virginia Prevention Research Center at the Robert C. Byrd Health Sciences Center at West Virginia University. We would also like to acknowledge study participants and the schools where data collection occurred.

These findings were also presented at the 12th Annual Hawaii International Conference on Education, Honolulu, Hawaii, January $5,2014$.

This work was supported by United States Department of Health and Human Services Centers for Disease Control and Prevention (U48 DPO00052) (Prevention Research Center Program); American Lung Association of Gulf Coast FL; FL Office of Tobacco Control; and the American Lung Association of New Jersey.

Jill A. Nolan, Ph.D., Assistant Professor of Health Education, Concord University.

Traci Jarrett, Ph.D., Project Director for the WV Prevention Research Center, West Virginia University.

Laura Kurth, Ph.D., National Institute of Occupational Safety and Health (NIOSH).

Kim Horn, Ed.D., Associate Dean of Research, Milken Institute School of Public Health, George Washington University.

Geri A. Dino, Ph.D., Director, WV Prevention Research Center, West Virginia University.
} 
through 12th graders reported ever trying smoking (CDC, 2010). Furthermore, about $20 \%$ of high school aged adolescents are current smokers, having smoked at least once in the past 30 days (CDC, 2010). These figures are alarming since early smoking initiation increases the likelihood of adult tobacco dependence (Chassin et al., 1996). Smokers also have poorer overall health status and spend more financially on medical care compared to non-smokers (CDC, 2010).

Another demonstrated predictor of health behavior is education. In general, higher levels of education show positive associations with health and increased life expectancy, and negative associations with health risks and health disparities (Freudenberg \& Ruglis, 2007). Of critical importance to the present study, research indicates a negative relationship between years of education attained and smoking among those over age 25 . Cutler, Deaton, and Lleras-Muney (2006) found that adults with more years of education were less likely to smoke (Cutler, Deaton, \& Lleras-Muney, 2006). Education also influences smoking cessation. Adults with lower education levels were less likely to have successful smoking cessation attempts (Chassin et al., 1996). With youth, the relationship between years of education attained and smoking status is difficult to examine because young people are still in the process of completing their schooling.

In contrast to educational attainment, the academic experience of youths still in school has potential for multi-dimensional examination of the relationship between substance use and education through other constructs such as academic performance, academic-related behaviors, and attitudes towards academics (Bryant et al., 2003). Low academic achievement in high school can have serious implications for high school completion and college matriculation because it is related to school dropout, and thus, educational attainment (Freudenberg \& Ruglis, 2007).

Cigarette use is negatively related to academic achievement among adolescents. In a national sample of 8th grade students, a low level of academic achievement, based on self-reported average letter grade, was associated with an increase in cigarette use over time. Students with high academic achievement were less likely than those with lower levels of achievement to use cigarettes or increase cigarette use over time (Bryant et al., 2003). High school juniors who reported smoking at least one cigarette in the past 30 days had a lower odds of completing high school and matriculating into a four year college compared to non-smokers (Cook \& Hutchinson, 2006). Students who smoke also have difficulties performing well in school and being in the school environment as a result of substance use behaviors (Bryant et al., 2003).

Other academic-related behaviors are also associated with substance use. This includes school misbehavior variables such as classes skipped, days of school skipped, school suspensions, and staying after school for misbehavior (Bryant et al., 2003). Students who are absent from school, with or without permission, are more likely to engage in health risk behaviors like smoking (Eaton, Brener, \& Kann, 2008). Studies exploring smoking intensity and academic performance have found that young adolescents who reported smoking daily or several times a week were more likely to report missing school than those who never smoked or were infrequent smokers (Alexander \& Klassen, 1988; Charlton \& Blair, 1989).

There is little research on associations among attitudes toward academic achievement and smoking. One study found that 8th grade students reporting higher cigarette use had lower levels of school bonding and felt less commitment to school and school effort (Bryant et al., 2003). Additionally, there is minimal knowledge on the relationship between academic achievement and smoking intensity among youths. Very little is known about how adolescent smokers perceive the importance of school and how these perceptions may be related to smoking intensity. The purpose of this study is to explore the relationship between perceived school importance 
and smoking intensity among high school students enrolled in a smoking cessation intervention. We hypothesized that higher levels of perceived academic importance would have a negative relationship with smoking intensity.

\section{Method}

\section{Participants}

Participants for this study included teens who voluntarily participated in the N-O-T school-based smoking cessation program (Horn et al., 2005). Participant data for the present investigation were derived from a larger, multi-state database of N-O-T studies spanning 1997 to $2009(N=8,855)$. Specifically, the analytic sample ( $N$ $=197$ ) included baseline data from participants from two studies in which data were available on two primary measures of interest: school importance and nicotine dependence. Consistently, participants were recruited for the enrollment across studies using flyers, school announcements, and word of mouth by N-O-T program facilitators (Massey et al., 2003). Inclusion criteria for the analytic sample included teens between the ages of 14-19, in the 9-12 grades, and who smoked at least one cigarette per day on weekdays/weekends. The study was approved by the West Virginia University Institutional Review Board.

\section{Procedures}

$\mathrm{N}-\mathrm{O}-\mathrm{T}$ is a 10 -session school-based smoking cessation program delivered by trained facilitators (Horn et al., 2005). N-O-T is a gender-tailored intervention specifically for teens and is delivered in same-gender groups of approximately 8-12 participants each led by same-gender facilitators. Program content and methods cover salient developmental issues for teens including peer pressure, coping skills, and decision making in addition to cessation strategies (Horn et al., 2005; Dino et al., 2001; Dino et al., 2004; Horn et al., 2004). N-O-T is a federally recognized SAMHSA (Substance Abuse and Mental Health Administration) evidence-based Model Program, a NCI (National Cancer Institute) Research Tested Intervention Program, and an OJJDP (Office of Juvenile Justice and Delinquency Prevention) Model Program. Studies on program effectiveness indicate end-of-program intent-to-treat quit rates between 15\% and 19\% (Horn et al., 2005) and cost-effectiveness analysis indicate N-O-T is a highly cost effective option for school-based tobacco intervention (compared to brief intervention) (Dino et al., 2008). All studies followed equivalent protocols.

\section{Measures}

Each participant was given a battery of standard assessment measures at baseline that assessed demographic information, smoking history, nicotine dependence, and school performance. The criterion variable, smoking intensity, measured average number of self-reported cigarettes smoked per day. Smoking intensity was created by combining two items that asked the number of self-reported cigarettes smoked per day Monday through Friday and the number of self-reported cigarettes smoked on Saturday and Sunday, then taking the average over 7 days.

$$
[(\# \text { cigarettes weekdays } * 5)+(\# \text { cigarettes Sat \& Sun *2)]/7 }
$$

Studies examining smoking intensity among adolescents have not consistently used a survey item or group of items to characterize smoking intensity. However, Eppel et al. (2006) conclude items querying number of cigarettes smoked per month have lower test- retest reliability, so measuring smoking intensity based on weekly self-reported cigarette use may be a more reliable measure of smoking intensity (Eppel et al., 2006). 
School Performance Measures Seven measures of perceived school performance were obtained from the baseline data collected from participants (See Table 1). A factor analysis with varimax rotation was performed and three of the measures of school performance "How important is regular school attendance to you?", "How important is it to you to get good grades?", and "How much did you enjoy attending school last semester?" factored together (See Table 1). The remaining perceived school performance measures did not factor together and were therefore not used in this study. The three school performance measures were combined to create a composite score for the underlying factor, perceived school importance, which ranged from 3 ("Not at all") to 15 ("Very important"). Additional predictors included age, grade, race, sex, and nicotine dependence. Nicotine dependence was measured using mFTQ (the teen Fagerstrom Tolerance Questionnaire) (Pomerleau et al., 1994), including seven items for a composite score ranging from 0-9 (with 0 being no dependence and 9 being substantial dependence). Race was dichotomized to form two categories, those who identified as whites and those who identified as any other race because there were so few non-white students in the final analytic sample.

\section{Statistical Analysis}

Data analyses were conducted using STATA 10.0IC (StataCorp, 2007). Initial variable exploration checked for percentage missing, normality, and outliers. OLS (Ordinary Least Squares) multiple regression models were tested. Missing data for each variable of interest was less than 3\% and were deleted listwise form the analysis. Post-hoc model fit tested Guass-Markov assumptions and model fit were assessed.

Table 1

Summary of Factor Loadings for Varimax Orthogonal Factor Rotation

\begin{tabular}{lccc}
\hline Item & Factor 1 & Factor 2 & Communality \\
\hline 1. About how many days were you absent last semester? & 0.181 & 0.197 & 0.072 \\
2. How important is regular school attendance to you? & 0.847 & 0.156 & 0.741 \\
3. On average, what kind of grades did you get last semester? & 0.169 & 0.760 & 0.606 \\
4. How important is it to you to get good grades? & 0.600 & 0.300 & 0.450 \\
5. How much did you enjoy attending school last semester? & 0.490 & 0.223 & 0.289 \\
6. How many clubs and activities are you involved in (e.g., football, art club, & 0.290 & 0.344 & 0.162 \\
student government, etc.)? & & 0.122 \\
7. Were you involved in any situations during the past marking period which \\
resulted in you receiving a disciplinary action (detention, suspension, etc.)? & -0.109 & -0.332 & 0.122 \\
\hline
\end{tabular}

\section{Results}

A total of 197 participants completed baseline evaluation assessing school importance and nicotine dependence with no missing data. Participants were primarily female (61.42\%) and Caucasian (89.34\%). Most students were in the 9 th $(30.46 \%)$ and 11 th $(29.95 \%)$ grades. Average smoking intensity was 11.67 cigarettes per day.

OLS multiple regression was performed between smoking intensity as the criterion variable and perceived school importance, nicotine dependence, race (white vs. non-white), grade, and gender as predictor variables. Assumptions were evaluated including normality, outliers, linearity, multicolinearity, and homoscedasticity. School importance had a slight negative skew that worsened with transformation. Therefore, the variable was not transformed. One outlier that exhibited significant influence on the other variables was not included in the final analysis. 
A significant regression equation was found $(F(5,191)=25.49, p<0.000)$, with an adjusted $R^{2}$ of 0.38 . The adjusted $R^{2}$ value of 0.38 indicates that the model accounts for more than a third of the variability of smoking intensity. School importance $t(191)=-2.96, p<0.003$, nicotine dependence $t(191)=9.84, p<0.000$, gender $t(191)=2.52, p<0.02$, and grade $t(191)=3.54, p<0.00$ were significant predictors of smoking intensity. Race $t(191)=0.437, p>0.50$ was not significant. Based on coefficient scores, for every unit increase in perceived school importance, there is a predicted 0.45 decrease in smoking intensity when controlling for covariates. Additionally, for every unit increase in nicotine dependence there is a 1.78 predicted increase in smoking intensity when controlling for covariates. Males displayed increased smoking intensity over females $\beta=1.82$. For every unit increase in grade, there is an expected 1.19 increase in smoking intensity (See Table 2).

Table 2

Predictors of Smoking Intensity

\begin{tabular}{llll}
\hline & $B$ & $S E b$ & $p$ \\
\hline School importance & -0.457 & 0.15 & 0.003 \\
Nicotine dependence & 1.78 & 0.18 & 0.000 \\
Race (white/not-white) & 0.883 & 1.13 & 0.437 \\
Grade & 1.19 & 3.54 & 0.000 \\
Gender & 1.82 & 0.721 & 0.013 \\
Constant & -4.67 & 4.00 & \\
$R^{2}=0.38$; adjusted $R^{2}=0.36$ & & & \\
\hline
\end{tabular}

\section{Discussion}

This cross-sectional study explored the relationship between perceived school importance and smoking intensity among a sample of smoking high school students seeking cessation. Consistent with previous N-O-T studies (Horn et al., 2003; Horn et al., 2008), nicotine dependence was the best predictor of smoking intensity at baseline among the variables in our model. However, the regression model in this study shows that even when controlling for nicotine dependence, perceived school importance is a significant predictor of smoking intensity. In addition, grade also influences a student's smoking intensity, although this result is expected due to maturation and increased use as teens age. Consistent with previous N-O-T studies (Branstetter et al., 2009), gender was also a significant predictor with males having higher levels of smoking intensity than females.

It should be noted that participants are unique among teen smokers due to enrollment in a cessation program (N-O-T). As predicted, perceived school importance has a significant influence on smoking intensity. This finding is noteworthy due to the established positive relationship between smoking and high school dropout (Mensch \& Kandel, 1988; Ellickson et al., 1998) and the established positive relationship between education and health (Freudenberg \& Ruglis, 2007). These findings could drive intervention efforts to both prevent or decrease smoking and promote education importance and performance. Due to the negative relationship between educational attainment and smoking (Cutler et al., 2006), increasing perceptions of school performance may be critical among teen smokers. Particularly with teens seeking cessation services, emphasizing the importance of academic performance could have a lasting impact on their success with cessation and health throughout the lifespan. 
Limitations include the fact that participants were all enrolled in a cessation program, which could bias their results. Therefore, the findings are not generalizable to all adolescent smokers. A more diverse sample including adolescent smokers not seeking cessation would be required to generalize results to a population beyond those students included in this study. Future studies should examine longitudinal school performance measures and smoking intensity among adolescent smokers pre- and post-intervention. Studies that monitor adolescents over time may provide insight into temporality and causality. Further studies may also be conducted to analyze changes in school performance measures and smoking intensity among adolescent smokers exposed to various smoking cessation interventions.

\section{References}

Alexander, C. S., \& Klassen, A. C. (1988). Drug use and illnesses among eighth grade students in rural schools. Public Health Reports (Washington, D.C.: 1974), 103(4), 394-399.

Branstetter, S. A., Horn, K., Dino, G., et al. (2009). Beyond quitting: Predictors of teen smoking cessation, reduction and acceleration following a school-based intervention. Drug and Alcohol Dependence, 99(1-3), 160-168.

Bryant, A. L., Schulenberg, J. E., O’Malley, P. M., et al. (2003). How academic achievement, attitudes, and behaviors relate to the course of substance use during adolescence: A 6-year, multiwave national longitudinal study. Journal of Research on Adolescence, 13(3), 361-397.

CDC. (2010). Trends in the prevalence of tobacco use. YRBSS.

Charlton, A., \& Blair, V. (1989). Absence from school related to children's and parental smoking habits. British Medical Journal, 298(6666), 90-92.

Chassin, L., Presson, C. C., Rose, J. S., et al. (1996). The natural history of cigarette smoking from adolescence to adulthood: Demographic predictors of continuity and change. Health Psychology: Official Journal of the Division of Health Psychology, American Psychological Association, 15(6), 478-484.

Cook, P., \& Hutchinson, R. (2006). Smoke signals: Adolescent smoking and school continuation. NBER Working Paper Series.

Cutler, D., Deaton, A., \& Lleras-Muney, A. (2006). The determinants of mortality. Journal of Economic Perspectives, 20(3), 97-120.

Dino, G., Horn, K., Goldcamp, J., et al. (2001). A 2-year efficacy study of Not On Tobacco in Florida: An overview of program successes in changing teen smoking behavior. Preventive Medicine, 33(6), 600-605.

Dino, G., Horn, K., Abdulkadri, A., et al. (2008). Cost-effectiveness analysis of the not on tobacco program for adolescent smoking cessation. Prevention Science, 9(1), 38-46.

Dino, G., Kamal, K., Horn, K., et al. (2004). Stage of change and smoking cessation outcomes among adolescents. Addictive Behaviors, 29(5), 935-940.

Eaton, D. K., Brener, N., \& Kann, L. K. (2008). Associations of health risk behaviors with school absenteeism: Does having permission for the absence make a difference? Journal of School Health, 78(4), 223-229.

Ellickson, P., Bui, K., Bell, R., et al. (1998). Does early drug use increase the risk of dropping out of high school? Journal of Drug Issues, 28(2), 357-380.

Eppel, A., O'Loughlin, J., Paradis, G., et al. (2006). Reliability of self-reports of cigarette use in novice smokers. Addictive Behaviors, 31(9), 1700-1704.

Ezzati, M., \& Lopez, A. D. (2003). Estimates of global mortality attributable to smoking in 2000. The Lancet, 362(9387), 847-852.

Freudenberg, N., \& Ruglis, J. (2007). Reframing school dropout as a public health issue. Preventing Chronic Disease, 4(4), A107-A107.

Horn, K., Dino, G., Kalsekar, I., et al. (2005). The impact of not on tobacco on teen smoking cessation: End-of-program evaluation results, 1998 to 2003. Journal of Adolescent Research, 20(6), 640-661.

Horn, K. A., Dino, G. A., Kalsekar, I. D. et al. (2004). Appalachian teen smokers: Not On Tobacco 15 months later. American Journal of Public Health, 94(2), 181-184.

Horn, K., Dino, G., Branstetter, S. A., et al. (2008). A profile of teen smokers who volunteered to participate in school-based smoking intervention. Tobacco Induced Diseases, 4, 6-6.

Horn, K., Fernandes, A., Dino, G., et al. (2003). Adolescent nicotine dependence and smoking cessation outcomes. Addictive Behaviors, 28(4), 769-776. 
Massey, C. J., Dino, G. A., Horn, K. A., et al. (2003). Recruitment barriers and successes of the American Lung Association's not-on-tobacco program. Journal of School Health, 73(2), 58-63.

Mensch, B. S., \& Kandel, D. B. (1988). Dropping out of high school and drug involvement. Sociology of Education, 61(2), 95-113.

Pomerleau, C., Carton, S., Lutzke, M., et al. (1994). Reliability of the fagerstrom tolerance questionnaire and the fagerstrom test for nicotine dependence. Addictive Behaviors, 19(1), 33-39.

StataCorp. (2007). Stata Statistical Software: Release 10. StataCorp LP: College Station, TX..

Warren, C. W., Jones, N. R., Eriksen, M. P., et al. (2006). Patterns of global tobacco use in young people and implications for future chronic disease burden in adults. The Lancet, 367(9512), 749-753. 